\title{
Dual-Source CT for Visualization of the Coronary Arteries in Heart Transplant Patients with High Heart Rates
}

Gorka Bastarrika ${ }^{1}$

Carlo Nicola De Cecco²

Maria Arraiza ${ }^{1}$

Stefano Mastrobuoni ${ }^{3}$

Jesús C. Pueyo ${ }^{1}$

Matias Ubilla ${ }^{3}$

Gregorio Rábago ${ }^{3}$

Keywords: coronary vessels, dual-source CT, heart transplantation, image quality, reconstruction interval

D0I:10.2214/AJR.07.3512

Received December 7, 2007; accepted after revision February 19, 2008.

'Department of Radiology, Clínica Universitaria, Universidad de Navarra, Ave. Pío XII, 36, 31008 Pamplona, Spain. Address correspondence to G. Bastarrika (bastarrika@unav.es).

${ }^{2}$ Department of Radiology, Universitá di Roma Sapienza-0spedale Sant'Andrea, Rome, Italy.

${ }^{3}$ Department of Cardiovascular Surgery, Clínica Universitaria, Universidad de Navarra, Pamplona, Spain.

AJR2008; 191:448-454

0361-803X/08/1912-448

() American Roentgen Ray Society

OBJECTIVE. The purpose of this study was to evaluate the quality of dual-source CT images of the coronary arteries in heart transplant recipients with high heart rates.

SUBJECTS AND METHODS. Contrast-enhanced dual-source CT coronary angiography was performed on 23 heart transplant recipients $(20$ men, three women; mean age, $61.1 \pm 12.8$ years). Data sets were reconstructed in 5\% steps from $30 \%$ to $80 \%$ of the R-R interval. Two blinded independent readers using a 5-point scale $(0$, not evaluative; 4 , excellent quality) assessed the quality of images of coronary segments.

RESULTS. The mean heart rate during scanning was $89.2 \pm 10.4$ beats/min. Interobserver agreement on the quality of images of the whole coronary tree was a kappa value of 0.78 and for selection of the optimal reconstruction interval was a kappa value of 0.82 . The optimal reconstruction interval was systole in 17 (74\%) of the 23 of heart transplant recipients. At the best reconstruction interval, diagnostic image quality (score $\geq 2$ ) was obtained in $92.1 \%$ (303 of 329) of the coronary artery segments. The mean image quality score for the whole coronary tree was $3.1 \pm 1.01$. No significant correlation between mean heart rate ( $\rho=0.31)$ or heart rate variability $(\rho=0.23)$ and overall image quality score was observed ( $p=$ not significant).

CONCLUSION. Dual-source CT acquisition yields coronary angiograms of diagnostic quality in heart transplant recipients. Mean heart rate and heart rate variability during scanning do not have a negative effect on the overall quality of images of the coronary arteries.

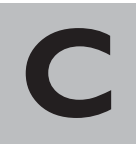

oronary allograft vasculopathy is a progressive disease that extensively affects the coronary arteries of a transplanted heart. This entity is a well-known factor limiting the successful long-term outcome of heart transplantation [1]. Coronary allograft vasculopathy is frequently asymptomatic, but when it occurs, the clinical manifestations vary from arrhythmia to congestive heart failure and even sudden cardiac death. In clinical routine, various noninvasive techniques are used for diagnosis, including exercise ECG, stress or exercise echocardiography, and radionuclide scintigraphy. Conventional coronary angiography remains the standard for ruling out coronary allograft vasculopathy. Intravascular sonography is the most sensitive tool for early detection. Because these techniques carry high cost and risk, especially in heart transplant recipients [2], a technique allowing noninvasive detection, characterization, and follow-up of coronary allograft vasculopathy is desirable.
Retrospectively ECG-gated MDCT has been found to be accurate in evaluation of the coronary arteries, to depict clinically significant coronary arterial stenosis, and to be useful in the assessment of vessel wall characteristics [3-7]. The usefulness of MDCT in the evaluation of coronary allograft vasculopathy in heart transplant recipients has been described [8-10]. However, because of factors such as high resting heart rate and postsurgical changes, imaging of heart transplant recipients continues to be a challenge in cardiac MDCT [11]. Limited temporal resolution of even 64-MDCT scanners with a 0.33 -second rotation time impedes widespread use of MDCT coronary angiography in this population. Premedication with $\beta$ blockers $[4,12,13]$ and use of multisegment reconstruction algorithms have been proposed for acquisition of high-quality cardiac images of patients with high heart rates [14, 15]. This approach, however, may not be sufficient for heart transplant recipients.

Dual-source CT has a high constant temporal resolution of 83 milliseconds independent 


\section{Dual-Source CT of Coronary Arteries}

of heart rate [16]. The scanner is characterized by two independent $\mathrm{x}$-ray tubes with corresponding detectors mounted on a rotating gantry with an angular offset of $90^{\circ}$ [16] Results of early investigations suggested that dual-source CT yields high-quality images of the coronary arteries at a wide range of heart rates without the use of $\beta$-blockers [17, 18]. Furthermore, according to one report [19], compared with invasive coronary angiography without heart rate control, dualsource CT has high diagnostic accuracy in the assessment of coronary artery disease even in a population with high pretest probability of having disease. To our knowledge, the feasibility of noninvasive dual-source CT coronary angiography in heart transplant recipients has not been systematically investigated. This study was performed to evaluate the quality of images of the coronary arteries at different reconstruction intervals and to assess the feasibility of high-quality noninvasive imaging of the coronary arteries in heart transplant recipients undergoing cardiac dual-source CT.

\section{Subjects and Methods Subjects}

Twenty-three heart transplant recipients who underwent coronary $\mathrm{CT}$ angiography to rule out coronary allograft vasculopathy were consecutively enrolled. Subjects with allergy to iodinated IV contrast media, impaired renal function (creatinine concentration $>1.2 \mathrm{mg} / \mathrm{dL}$ ), and arrhythmia or frequent extrasystole were excluded. No oral or IV $\beta$-blocker premedication to lower or stabilize the heart rate was indicated before imaging. Two minutes before $\mathrm{CT}$, all patients received a tablet of $0.4 \mathrm{mg}$ of sublingual nitroglycerin (Vernies, Pfizer). The study protocol was approved by our institutional review board, and written informed consent was obtained from all patients.

\section{Dual-Source CT Image Acquisition}

A dual-source CT scanner (Somatom Definition, Siemens Medical Solutions) was used to perform cardiac CT examinations. Scans were acquired at end inspiration. Acquisition was craniocaudal starting cranial to the origin of the coronary arteries and ending caudal to the dome of the diaphragm. Patients were placed centrally in the scanner to ensure adequate coverage of the whole heart by the two tubes. Tube voltage of $120 \mathrm{kV}$ and effective current of $350 \mathrm{mAs} /$ rotation for both tubes (CT volume dose index, $41 \mathrm{mGy}$ ) were used with automatic tube current modulation (ECG pulsing). Full tube current was administered between $30 \%$ and $80 \%$ of the cardiac cycle. Other dual-source
CT parameters were as follows: gantry rotation time, 0.33 milliseconds; detector collimation, $32 \times 0.6 \mathrm{~mm}$; slice acquisition, $64 \times 0.6 \mathrm{~mm}$ with a $z$-flying focal spot [20]. The pitch was variable $(0.2-0.35)$ and automatically adapted to heart rate. Retrospectively ECG-gated coronary dual-source $\mathrm{CT}$ angiography was performed after IV injection of $70 \mathrm{~mL}$ of contrast medium (iomeprol $400 \mathrm{mg} \mathrm{I} /$ $\mathrm{mL}$, Iomeron 400, Bracco) through an antecubital vein. A dual-head power injector (CT Stellant, Medrad) was used for continuous infusion of the contrast agent at a flow rate of $5 \mathrm{~mL} / \mathrm{s}$ followed by a $50-\mathrm{mL}$ saline flush chaser. A region of interest was placed in the ascending aorta for data acquisition. Scanning started automatically 6 seconds after the attenuation reached a predefined threshold of $100 \mathrm{H}$. Mean scanning time was 8 seconds.

\section{Dual-Source CT Image Reconstruction}

Dual-source CT data were retrospectively reconstructed with a monosegment reconstruction algorithm. In dual-source $\mathrm{CT}$ this algorithm operates with data from $90^{\circ}$ rotation of both detectors to generate images [16]. The field of view was manually adjusted for each patient to cover the whole heart. A dedicated medium soft-tissue convolution kernel (B26f) was chosen for image reconstruction. Dual-source CT data sets were reconstructed with a slice thickness of $0.75 \mathrm{~mm}$ and $0.4-\mathrm{mm}$ reconstruction increment in 5\% steps of the R-R interval within the full tube current window (30-80\%). No manual ECG editing was necessary because of the low variability in heart rates. Images obtained were transferred to an external workstation (Leonardo, Siemens Medical Solutions) equipped with a dedicated cardiac postprocessing software tool (Syngo Circulation II, Siemens Medical Solutions). Axial source images, multiplanar reconstructions, 3-mm thin-slab maximum-intensity-projection reconstructions, volumerendered images, and curved multiplanar reformations were used for image evaluation.

\section{Dual-Source CT Data Analysis}

Two independent experienced readers blinded to patient information, heart rate, and ECG results assessed the diagnostic quality of images of the coronary artery segments. The segments were evaluated according to the 16-segment classification of the American Heart Association [21]. The right coronary artery (RCA) included segments $1-4$, the left main coronary artery (LCA) and left anterior descending (LAD) artery included segments 5-10, and the left circumflex artery (LCX) included segments 11-15. If present, the intermediate artery was segment 16 . Analysis was performed on all coronary segments with a luminal diameter of at least $1 \mathrm{~mm}$ [22].
For each coronary segment, image quality was graded on a 5-point scale as follows: 0 , not evaluative, vessels not differentiable; 1 , not assessable owing to blurring and motion artifact impeding arterial evaluation; 2 , sufficient diagnostic quality to rule out significant coronary artery disease; 3 , minor artifacts but good quality for ruling out coronary artery disease and for grading coronary stenosis; 4, excellent quality for accurate evaluation of coronary artery disease and characterization of atherosclerotic plaques (Fig. 1). The system used to score the average quality of coronary artery segments was adapted from a previous report [23]. Quality scores of 2 or greater were considered acceptable for routine clinical diagnosis (Fig. 2). Heart rate variability was calculated as SD from the average heart rate, as previously reported [23]. Interobserver concordance for quality grading of coronary artery segments was evaluated. The influence of mean heart rate and heart rate variability on the mean diagnostic quality of images of each coronary segment and coronary artery (LCA, LAD, LCX, RCA) was analyzed.

\section{Statistical Analysis}

Quantitative variables of image quality were expressed as mean $\pm \mathrm{SD}$. Interobserver agreement for image quality and best reconstruction interval was calculated with Cohen kappa statistics [24] and interpreted as follows: less than 0.20 , slight or poor agreement; $0.20-0.40$, fair agreement; $0.41-$ 0.80 , moderate agreement; greater than 0.80 , excellent agreement. Spearman's rank correlation coefficient was performed to compare image quality scores for all coronary segments in each patient with mean heart rate and heart rate variability during the examination. Multivariate regression analysis was performed to evaluate the coeffects of mean heart rate and heart rate variability on mean image quality score per subject. A level of $p<0.05$ indicated a statistically significant difference. Data analysis was performed with commercially available statistical software (MedCalc, version 9.3.0.0, MedCalc; SPSS for Windows, version 14.1, SPSS).

\section{Results}

Dual-source CT coronary angiography was successfully performed within a single breath-hold without complications for all orthotopic heart transplant recipients. All examinations were diagnostic. The mean age of the 23 subjects ( 20 men, three women) in the study was $61.1 \pm 12.8$ years (range, 25-77 years). Mean time from heart transplantation was $111.1 \pm 72.7$ months (range, 12-245 months). There was no evidence of allograft rejection at the time of dual-source CT. 


\section{Bastarrika et al.}
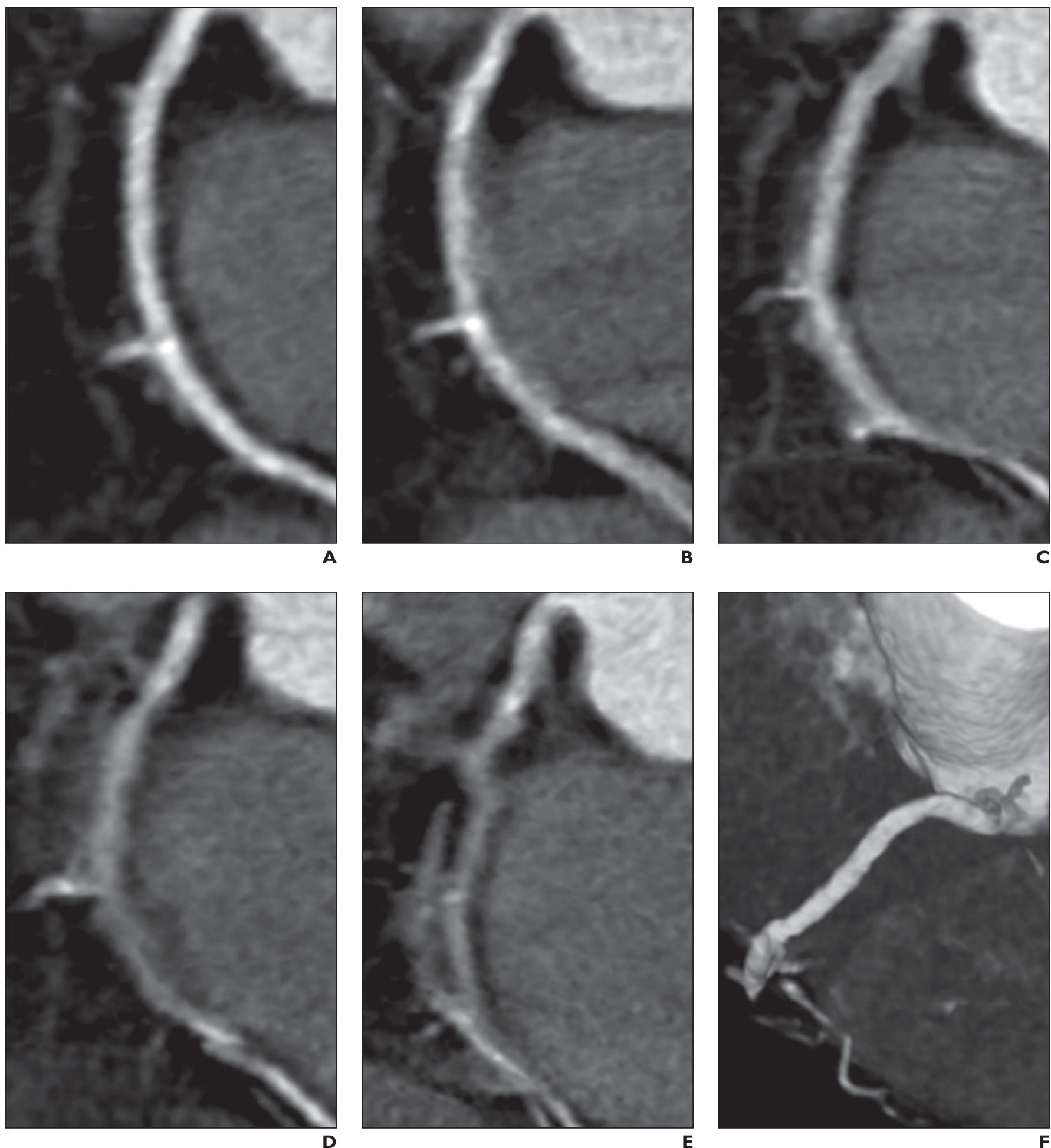

Fig. 1-69-year-old heart transplant recipient with heart rate of 90 beats/min who underwent dual-source CT coronary angiography. Reconstructions of segment 2 of right coronary artery in different phases of cardiac cycle show examples of 5-point grading scale. Quality scores of 2 or greater were considered acceptable for routine clinical diagnosis.

A, Score 4 image is of excellent quality for accurate evaluation of coronary artery disease.

B, Score 3 image has minor artifacts and is of good quality for ruling out coronary artery disease.

C. Score 2 image is of sufficient diagnostic quality for ruling out significant coronary artery disease.

D, Score 1 image is not assessable owing to blurring and motion artifact impeding arterial evaluation.

E, Score 0 image is not evaluative. Vessels are not differentiable.

F, Volume-rendered reconstruction shows best reconstruction interval for this coronary segment. 


\section{Dual-Source CT of Coronary Arteries}
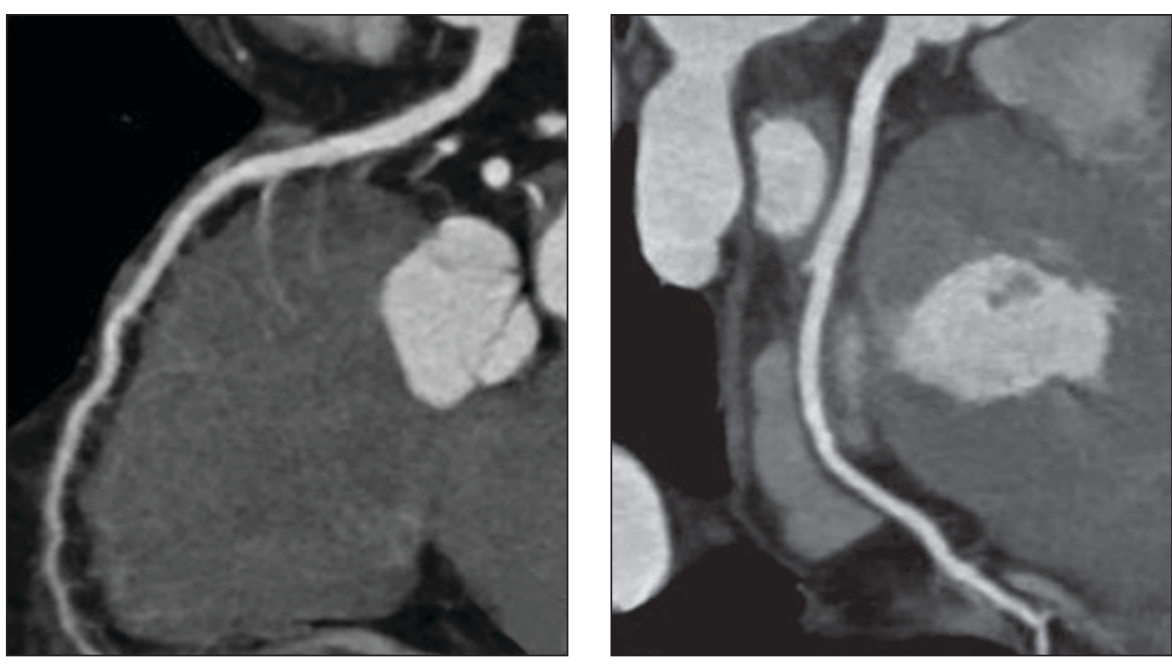

A
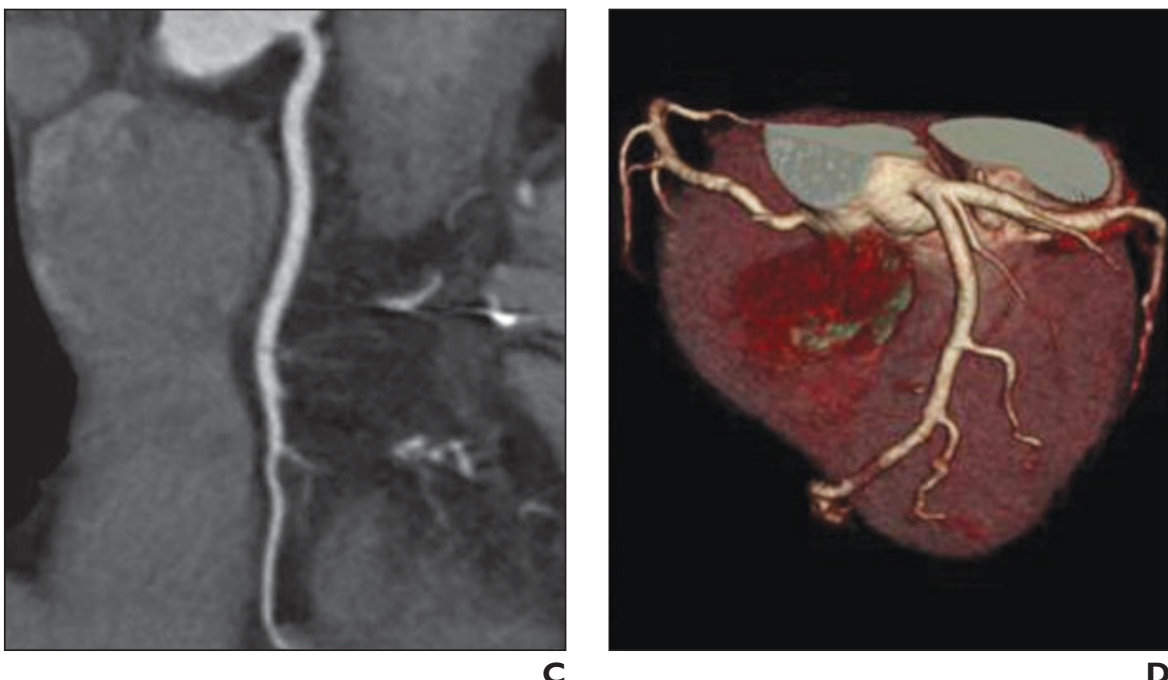

\section{Quality of Coronary Artery Images}

A total of 329 coronary artery segments in 23 patients were available for evaluation. Sixteen segments were missing owing to anatomic variations (10 segments) or marked thinning $(<1 \mathrm{~mm})$ of the vessels (six segments). Each coronary segment was evaluated in a $5 \%$ increment of the R-R interval within the full tube current window (30-80\% of the R-R interval, 11 phases), resulting in a total of 3,619 time points. The optimal reconstruction interval was systole in $17(74 \%)$ of the 23 heart transplant recipients. The optimal systolic reconstruction interval was $45 \%$ of the R-R interval in seven (41\%), 35\% of the R-R interval in five (29\%), $40 \%$ of the $\mathrm{R}-\mathrm{R}$ interval in four $(24 \%)$, and $30 \%$ of the $\mathrm{R}-\mathrm{R}$ interval in one $(6 \%)$ of the 17 subjects. The best image quality scores were obtained with diastolic reconstructions in six subjects
Fig. 2-68-year-old man with mean heart rate of 87 beats/min and heart rate variability of 2.26 beats/min. Despite high heart rate during scanning, dual-source CT coronary angiograms of all coronary arteries are of diagnostic quality.

A-C, Curved multiplanar reconstructions of left anterior descending artery $(\mathbf{A})$, right coronary artery (B), and circumflex artery (C).

D, Volume-rendered image of whole coronary tree (D)

$2.37 \pm 0.44$ was obtained for the LCA, $1.90 \pm 0.55$ for the LCX, and $2.12 \pm 0.62$ for the RCA. The scores at the best reconstruction interval are shown in Table 1. Overall interobserver agreement on image quality ratings for the whole coronary tree was a kappa value of 0.78 . The kappa value was 0.74 for the LCA, 0.79 for the LCX, and 0.80 for the RCA. Interobserver agreement for selection of the optimal reconstruction interval for the coronary segments was excellent $(\kappa=0.82)$.

Effect of Mean Heart Rate and Heart Rate Variability on Quality of Coronary Artery Images

The mean heart rate during dual-source CT was $89.2 \pm 10.4$ beats/min (range, 71-108 beats/min). Despite the high resting heart rates, diagnostic image quality was obtained for the heart transplant recipients. Overall, no significant correlation was found between average heart rate and mean quality score for all coronary segments $(\rho=0.31, p=0.16)$. This correlation also was not significant in per-vessel analysis for the LCA $(\rho=0.17$, $p=0.44), \operatorname{LCX}(\rho=0.21, p=0.34)$, or RCA ( $\rho=0.37, p=0.09$ ) (Fig. 3). Thus mean quality scores were independent of heart rate over the range of heart rates studied.

The mean heart rate variability during cardiac dual-source CT examinations was $3.2 \pm$ 3.2 beats/min (range, $1.4-17.6$ beats/min). No significant correlation was found between average heart rate variability and mean quality score for all coronary segments in each patient ( $\rho=0.23, p=0.29)$. This correlation was not significant in per-vessel analysis for the LCA $(\rho=0.17, p=0.43), \operatorname{LCX}(\rho=0.20$, $p=0.36)$, or RCA $(\rho=0.16, p=0.47)$. Multivariate regression analysis showed no significant coeffect of mean heart rate or heart rate variability on overall coronary image quality per patient $(r=0.48, p=0.07)$.

\section{Discussion}

Development of coronary allograft vasculopathy continues to be a major cause of mortality among heart transplant recipients 
Bastarrika et al.

TABLE I: Demographic Data and Results for Heart Transplant Recipients Undergoing Dual-Source CT of the Coronary Arteries

\begin{tabular}{l|c}
\hline \multicolumn{1}{c|}{ Characteristic } & Value \\
\hline No. of patients & 23 \\
Sex ratio (M/F) & $20 / 3$ \\
Age (y) & $61.1 \pm 12.8(25-77)$ \\
Time from transplantation (mo) & $111.1 \pm 72.7(12-245)$ \\
Mean heart rate (beats/min) & $89.2 \pm 10.4(71-108)$ \\
Mean heart rate variability (beats/min) & $3.2 \pm 3.2(1.4-17.6)$ \\
Image score at best reconstruction interval & \\
Whole coronary tree & $3.1 \pm 1.01$ \\
Left coronary artery & $3.18 \pm 0.98$ \\
Circumflex artery & $2.97 \pm 1.12$ \\
Right coronary artery & $3.09 \pm 0.93$ \\
\hline
\end{tabular}

Note-Data are mean $\pm S D$ and range.

[25]. Ischemia usually is silent until the disease is advanced [26]. Conventional coronary angiography with or without intravascular sonography is the reference standard for the diagnosis [27]. The presence of coronary allograft vasculopathy detected with intravascular sonography has been found predictive of cardiac events [28] and long-term morbidity and mortality [29]. Nevertheless, especially for heart transplant recipients, conventional coronary angiography and intravascular sonography carry a small but considerable risk of complications such as arrhythmia, coronary dissection, and thrombosis [30-32].
MDCT has been found $[8,10]$ to yield goodto excellent-quality images of the coronary arteries, to depict the coronary artery lumen and wall, and to depict occlusive coronary disease in heart transplant recipients. Furthermore, the results of a study with a selected cohort of heart transplant patients [9] emphasized the potential of MDCT for replacing conventional coronary angiography. However, the diagnostic accuracy of MDCT coronary angiography depends mainly on image quality, a variable that relies heavily on heart rate and the timing of reconstruction [33]. Even with 64-MDCT scanners with a 0.33-second rotation time, limited temporal resolution remains a problem. Results continue to show that image quality is limited at certain heart rates [5] and that $\beta$-blocker therapy before scanning can lower and stabilize the heart rate [5, 23]. However, the safety of $\beta$-blocker premedication of heart transplant recipients is unclear [34]. This inconvenience seems to be overcome with dual-source CT. Our results indicate that with dual-source CT, diagnostic-quality images of all coronary arteries can be achieved without $\beta$-blockers in heart transplant recipients despite their high rest heart rates.
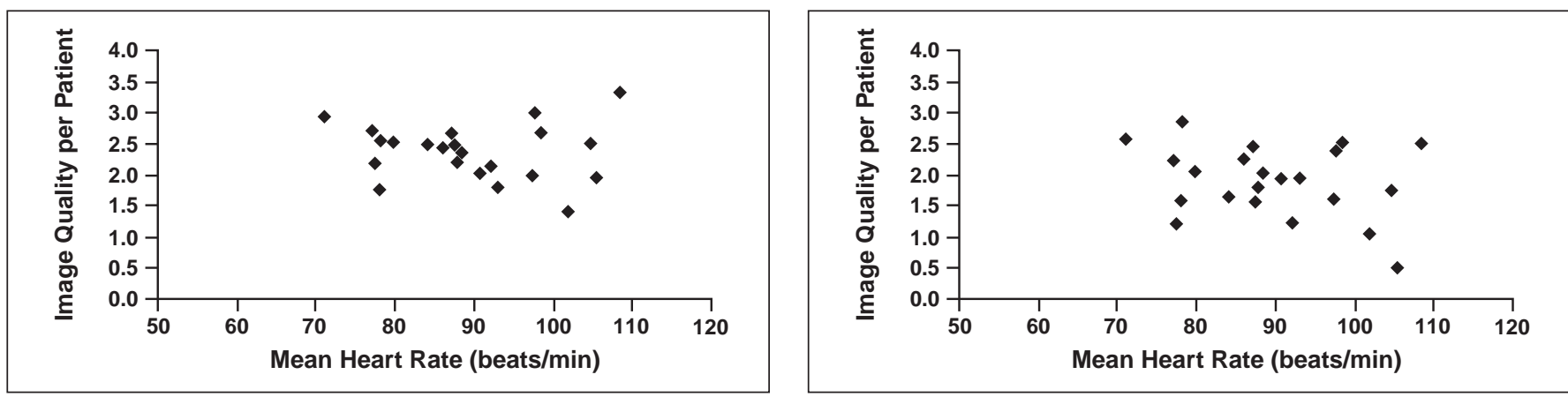

A
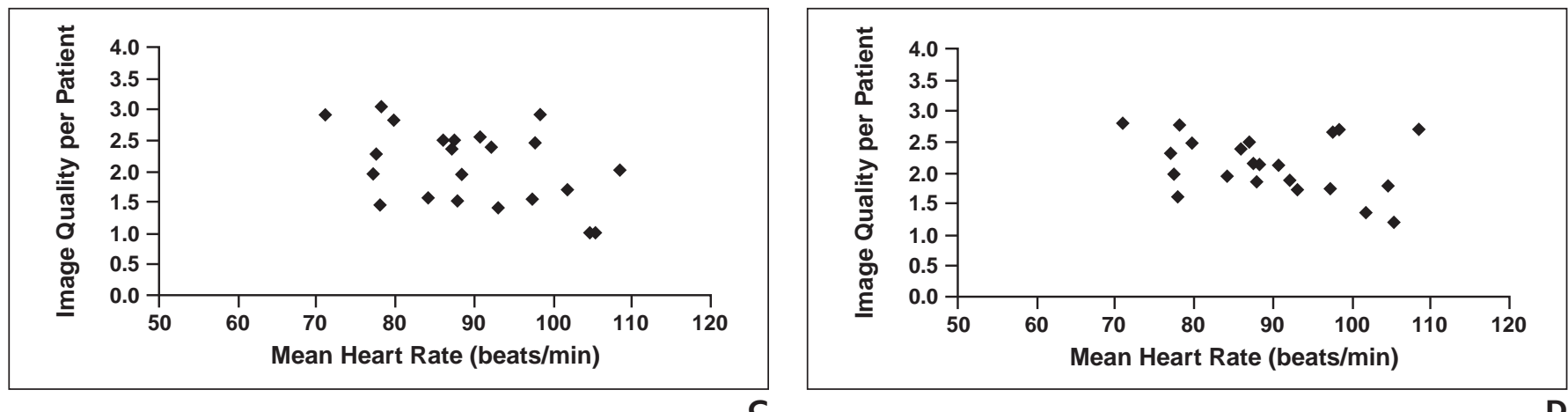

Fig. 3-Dispersion plots of mean image quality scores in relation to mean heart rate for all coronary segments in each patient.

A, Dispersion plot shows results for left coronary artery image quality.

B, Dispersion plot shows results for right coronary artery image quality.

C, Dispersion plot shows results for circumflex artery image quality.

D, Dispersion plot shows results for overall image quality. 


\section{Dual-Source CT of Coronary Arteries}

A significant negative correlation between overall quality of images of the coronary arteries and mean heart rate during scanning has been consistently reported since the introduction of MDCT cardiac imaging in clinical practice $[5,35]$. However, studies of the use of 64-MDCT with a 0.33 -second rotation time and dual-source technique have shown no significant correlation between image quality and heart rate $[22,23]$. This lack of correlation may be due to the increased temporal resolution, decreased total scanning time, and improved spatial resolution of 64-MDCT scanners [36]. In particular, the increased 83-millisecond temporal resolution of dual-source CT with respect to the 165 milliseconds of 64-MDCT allows imaging of the coronary arteries with excellent quality even at high resting heart rates. In our series, when all evaluated R-R intervals were analyzed, diagnostic image quality (score $\geq 2$ ) was found in $68.7 \%$ of all coronary segments (score 4 in $16.1 \%$, score 3 in $25.4 \%$, score 2 in $27.2 \%$ ). However, when the best reconstruction interval was considered, we found excellent quality in $44.4 \%$ of coronary segments with minor artifacts in $29.2 \%$ of coronary segments and moderate artifacts in $18.5 \%$ of coronary segments.

Our study results confirm the absence of negative correlation between overall quality of coronary artery images and mean heart rate in cardiac dual-source $\mathrm{CT}$ examinations performed on a population with characteristically high resting heart rates. Overall analysis of the coronary tree and per-vessel analysis both revealed no negative effect of mean heart rate during dual-source $\mathrm{CT}$ on the quality of images of the coronary arteries. This finding emphasizes the robustness of the technique, which allows excellent image quality independent of heart rate.

On the other hand, heart rate variability has been consistently considered a major determinant of image quality in noninvasive coronary imaging. Nonproportional shortening and prolongation of the cardiac phases occurs at differing heart rates [37]. Therefore, at imaging of subjects with highly variable heart rates, images are not generated in precisely the same cardiac phase. As a consequence, especially at high resting heart rates, artifacts that can hamper the diagnostic quality of images of the coronary arteries can occur $[5,23,38]$. This problem may be partially solved with use of a multisegment reconstruction algorithm for data reconstruction [38]. Use of such algorithms, however, increases image blurring $[23,38]$.
The effect of heart rate variability on the quality of coronary artery images has been systemically analyzed by Leschka et al. [23] using 64-MDCT and Matt et al. [22] using dual-source CT. Matt et al. did not find a significant effect of interheartbeat variation on overall image quality. According to those authors, the absence of negative correlation was probably due to the use of a monosegment reconstruction algorithm for dual-source CT examinations. When, however, Matt et al. analyzed the coeffects of mean heart rate and heart rate variability on overall diagnostic quality of images of the coronary arteries, a significant coeffect was found, emphasizing the negative contribution of heart rate variability to mean quality of coronary images.

Our results indicate that in the case of heart transplant recipients, the heart rate variability that can occur during coronary dual-source CT does not have a negative effect on overall diagnostic image quality. Correlation between the image quality for individual coronary vessels and heart rate variability also was not significant in per-vessel analysis for each subject. Furthermore, unlike Matt et al. [22], we found no significant coeffect of mean heart rate and heart rate variability on overall diagnostic quality of coronary images. This observation reflects the unique cardiac physiologic state after heart transplantation. In heart transplant recipients, sympathetic and parasympathetic innervations are impaired. As a consequence, heart rate does not vary according to respiration and does not respond to Valsalva maneuvers. The sympathetic input with positive chronotropic effect that commonly occurs after rapid IV contrast injection in patients who have not received a heart transplant is inhibited in heart transplant recipients. Consequently, heart rate variability during dual-source CT may be less in heart transplant recipients than in patients who have not undergone transplantation and therefore seems not to affect the diagnostic image quality of noninvasive coronary angiograms.

This study had several limitations. First, the study population was a small number of heart transplant recipients. The study was developed to determine the feasibility of noninvasive coronary imaging with dualsource CT in heart transplant recipients without heart rate control. Even though the quality-scoring method was subjective (scoring influenced by subjectivity bias), the very good interobserver agreement (overall image quality rating, $\kappa=0.78$; selection of optimal reconstruction interval, $\kappa=0.82$ ) suggests that coronary imaging with high diagnostic quality is feasible with dual-source CT. No direct comparison with invasive coronary angiography or with intravascular sonography was performed to assess the effect of image quality on the utility of dual-source CT in accurate detection and quantification of coronary artery disease. The referring physicians refused to request conventional coronary angiography after normal findings were made at dual-source CT.

Another limitation was that evaluation of dual-source CT coronary angiograms was limited to vessels with a diameter greater than $1 \mathrm{~mm}$. As a consequence, small-vessel involvement might not have been evaluated with dual-source CT. Nevertheless, coronary lesions would not have been accessible to percutaneous revascularization owing to the small size of the vessels. Finally, this study relied on the half-scan reconstruction algorithm. In 64-MDCT scanners, use of twosegment reconstruction has been described as improving the image quality of MDCT coronary angiography [39]. The role of this reconstruction algorithm for increasing the temporal resolution of dual-source CT requires additional analysis.

Dual-source CT is reliable for noninvasive acquisition of coronary angiograms of diagnostic quality in heart transplant recipients despite the high resting heart rates of these patients.

\section{References}

1. Taylor DO, Edwards LB, Boucek MM, Trulock EP, Keck BM, Hertz MI. The registry of the International Society for Heart and Lung Transplantation: twenty-first official adult heart transplant report-2004. J Heart Lung Transplant 2004; 23:796-803

2. Clague JR, Cox ID, Murday AJ, Charokopos N, Madden BP. Low clinical utility of routine angiographic surveillance in the detection and management of cardiac allograft vasculopathy in transplant recipients. Clin Cardiol 2001; 24:459-462

3. Leschka S, Alkadhi H, Plass A, et al. Accuracy of MSCT coronary angiography with 64-slice technology: first experience. Eur Heart J 2005; 26:1482-1487

4. Raff GL, Gallagher MJ, O'Neill WW, Goldstein JA. Diagnostic accuracy of noninvasive coronary angiography using 64-slice spiral computed tomography. J Am Coll Cardiol 2005; 46:552-557

5. Wintersperger BJ, Nikolaou K, von Ziegler F, et al. Image quality, motion artifacts, and reconstruction timing of 64-slice coronary computed tomography angiography with 0.33 -second rotation speed. Invest Radiol 2006; 41:436-442

6. Leber AW, Knez A, Becker A, et al. Accuracy of 


\section{Bastarrika et al.}

multidetector spiral computed tomography in identifying and differentiating the composition of coronary atherosclerotic plaques: a comparative study with intracoronary ultrasound. $J$ Am Coll Cardiol 2004; 43:1241-1247

7. Achenbach S, Moselewski F, Ropers D, et al. Detection of calcified and noncalcified coronary atherosclerotic plaque by contrast-enhanced, submillimetermultidetectorspiral computed tomography: a segment-based comparison with intravascular ultrasound. Circulation 2004; 109:14-17

8. Sigurdsson G, Carrascosa P, Yamani MH, et al. Detection of transplant coronary artery disease using multidetector computed tomography with adaptative multisegment reconstruction. $J \mathrm{Am}$ Coll Cardiol 2006; 48:772-778

9. Romeo G, Houyel L, Angel CY, Brenot P, Riou JY, Paul JF. Coronary stenosis detection by 16-slice computed tomography in heart transplant patients: comparison with conventional angiography and impact on clinical management. $J \mathrm{Am}$ Coll Cardiol 2005; 45:1826-1831

10. Gregory SA, Ferencik M, Achenbach S, et al. Comparison of sixty-four-slice multidetector computed tomographic coronary angiography to coronary angiography with intravascular ultrasound for the detection of transplant vasculopathy. Am J Cardiol 2006; 98:877-884

11. Ferencik M, Gregory SA, Butler J, et al. Analysis of cardiac dimensions, mass and function in heart transplant recipients using 64-slice multi-detector computed tomography. J Heart Lung Transplant 2007; 26:478-484

12. Nikolaou K, Knez A, Rist C, et al. Accuracy of 64-MDCT in the diagnosis of ischemic heart disease. AJR 2006; 187:111-117

13. Leber AW, Knez A, von Ziegler F, et al. Quantification of obstructive and nonobstructive coronary lesions by 64 -slice computed tomography: a comparative study with quantitative coronary angiography and intravascular ultrasound. $J$ Am Coll Cardiol 2005; 46:147-154

14. Schlosser T, Mohrs OK, Magedanz A, Voigtlander T, Schmermund A, Barkhausen J. Assessment of left ventricular function and mass in patients undergoing computed tomography (CT) coronary angiography using 64-detector-row CT: comparison to magnetic resonance imaging. Acta Radiol 2007; 48:30-35

15. Schlosser T, Pagonidis K, Herborn CU, et al. Assessment of left ventricular parameters using 16-MDCT and new software for endocardial and epicardial border delineation. AJR 2005; 184:765-773

16. Flohr TG, McCollough CH, Bruder H, et al. First performance evaluation of a dual-source CT (DSCT) system. Eur Radiol 2006; 16:256-268

17. Johnson TR, Nikolaou K, Wintersperger BJ, et al. Dual-source CT cardiac imaging: initial experience. Eur Radiol 2006; 16:1409-1415

18. Achenbach S, Ropers D, Kuettner A, et al. Contrast-enhanced coronary artery visualization by dual-source computed tomography: initial experience. Eur J Radiol 2006; 57:331-335

19. Scheffel H, Alkadhi H, Plass A, et al. Accuracy of dual-source CT coronary angiography: first experience in a high pre-test probability population without heart rate control. Eur Radiol 2006; 16: 2739-2747

20. Flohr TG, Stierstorfer K, Ulzheimer S, Bruder H, Primak AN, McCollough $\mathrm{CH}$. Image reconstruction and image quality evaluation for a 64-slice CT scanner with z-flying focal spot. Med Phys 2005; 32:2536-2547

21. Austen WG, Edwards JE, Frye RL, et al. A reporting system on patients evaluated for coronary artery disease: report of the Ad Hoc Committee for Grading of Coronary Artery Disease, Council on Cardiovascular Surgery, American Heart Association. Circulation 1975; 51[4 suppl]:5-40

22. Matt D, Scheffel H, Leschka S, et al. Dual-source CT coronary angiography: image quality, mean heart rate, and heart rate variability. AJR 2007; 189:567-573

23. Leschka S, Wildermuth S, Boehm T, et al. Noninvasive coronary angiography with 64-section CT: effect of average heart rate and heart rate variability on image quality. Radiology 2006; 241: 378-385

24. Cohen J. A coefficient of agreement for nominal scales. Educ Psychol Meas 1960; 20:37-46

25. Miller LW, Schlant RC, Kobashigawa J, Kubo S, Renlund DG. 24th Bethesda conference: cardiac transplantation-task force 5; complications. $J$ Am Coll Cardiol 1993; 22:41-54

26. Stark RP, McGinn AL, Wilson RF. Chest pain in cardiac-transplant recipients: evidence of sensory reinnervation after cardiac transplantation. $N$ Engl J Med 1991; 324:1791-1794

27. Behrendt D, Ganz P, Fang JC. Cardiac allograft vasculopathy. Curr Opin Cardiol 2000; 15:422- 429

28. Mehra MR, Ventura HO, Stapleton DD, Smart FW, Collins TC, Ramee SR. Presence of severe intimal thickening by intravascular ultrasonography predicts cardiac events in cardiac allograft vasculopathy. J Heart Lung Transplant 1995; 14:632-639
29. Tuzcu EM, Kapadia SR, Sachar R, et al. Intravascular ultrasound evidence of angiographically silent progression in coronary atherosclerosis predicts long-term morbidity and mortality after cardiac transplantation. J Am Coll Cardiol 2005; 45:1538-1542

30. Tsutsui H, Ziada KM, Schoenhagen P, et al. Lumen loss in transplant coronary artery disease is a biphasic process involving early intimal thickening and late constrictive remodeling: results from a 5-year serial intravascular ultrasound study. Circulation 2001; 104:653-657

31. St. Goar FG, Pinto FJ, Alderman EL, et al. Intracoronary ultrasound in cardiac transplant recipients: in vivo evidence of "angiographically silent" intimal thickening. Circulation 1992; 85:979-987

32. Bashore TM, Bates ER, Berger PB, et al. American College of Cardiology/Society for Cardiac Angiography and Interventions Clinical Expert Consensus Document on Cardiac Catheterization Laboratory Standards: a report of the American College of Cardiology Task Force on Clinical Expert Consensus Documents. J Am Coll Cardiol 2001; 37:2170-2214

33. Herzog C, Abolmaali N, Balzer JO, et al. Heartrate-adapted image reconstruction in multidetector-row cardiac CT: influence of physiological and technical prerequisite on image quality. Eur Radiol 2002; 12:2670-2678

34. Bae KT, Hong C, Takahashi N, et al. Multi-detector row computed tomographic angiography in pediatric heart transplant recipients: initial observations. Transplantation 2004; 77:599-602

35. Hoffmann MH, Shi H, Manzke R, et al. Noninvasive coronary angiography with 16-detector row CT: effect of heart rate. Radiology 2005; 234:86-97

36. Flohr T, Stierstorfer K, Raupach R, Ulzheimer S, Bruder H. Performance evaluation of a 64-slice CT system with z-flying focal spot. Rofo 2004; 176:1803-1810

37. Kovacs SJ Jr. The duration of the QT interval as a function of heart rate: a derivation based on physical principles and a comparison to measured values. Am Heart J 1985; 110:872-878

38. Flohr T, Ohnesorge B. Heart rate adaptive optimization of spatial and temporal resolution for electrocardiogram-gated multislice spiral CT of the heart. J Comput Assist Tomogr 2001; 25:907-923

39. Herzog C, Nguyen SA, Savino G, et al. Does twosegment image reconstruction at 64-section CT coronary angiography improve image quality and diagnostic accuracy? Radiology 2007; 244:121-129 